See discussions, stats, and author profiles for this publication at: https://www.researchgate.net/publication/327238004

\title{
Effect of ionic strength on the structure and elongational kinetics of vimentin filaments
}

Article in Soft Matter · August 2018

DOI: 10.1039/C8SM01007B

CITATIONS

8 authors, including:

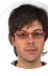

Carlos G. Lopez

RWTH Aachen University

52 PUBLICATIONS 123 CITATIONS

SEE PROFILE

Anders Aufderhorst-Roberts

AMOLF

11 PUBLICATIONS 73 CITATIONS

SEE PROFILE

Some of the authors of this publication are also working on these related projects:

Project Polyelectrolyte structure and solution rheology. View project

Project Combining Microfluidics with Small Angle Neutron Scattering View project
READS

48

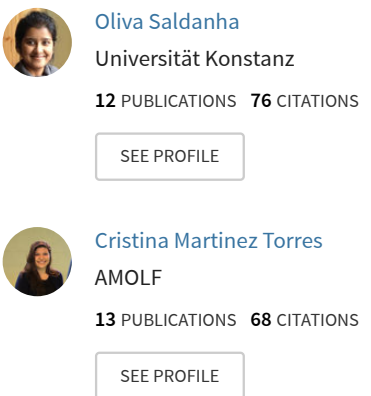




\title{
Journal Name
}

\section{ARTICLE TYPE}

Cite this: DOI: $10.1039 / x x x x x x x x x x$

\section{Effect of ionic strength on the structure and elonga- tional kinetics of vimentin filaments ${ }^{\dagger}$}

\author{
Carlos G. Lopez ${ }^{a, 末}$, Oliva Saldanha, ${ }^{b}$ Anders Aufderhorst-Roberts ${ }^{c}$, Cristina Martinez- \\ Torres $^{c}$, Merel Kuijs ${ }^{c}$, Gijsje H. Koenderink ${ }^{c}$ Sarah Köster, ${ }^{b *}$ Klaus Huber $^{a *}$
}

Received Date

Accepted Date

DOI: $10.1039 / x x x x x x x x x x$

www.rsc.org/journalname
Intermediate filaments are a major structural element in the cytoskeleton of animal cells that mechanically integrate other cytoskeletal components and absorb externally applied stress. Their role is likely to be linked to their complex molecular architecture which is the product of a multi-step assembly pathway. Intermediate filaments form tetrameric subunits which assemble in the presence of monovalent salts to form unit length filaments that subsequently elongate by end-to-end annealing. The present work characterises this complex assembly process using reconstituted vimentin intermediate filaments with monovalent salts as an assembly trigger. A multi-scale approach is used, comprising static light scattering, dynamic light scattering and quantitative scanning transmission electron microscopy (STEM) mass measurements. Light scattering reveals the radius of gyration $\left(R_{g}\right)$, molecular weight $\left(M_{w}\right)$ and diffusion coefficient $(D)$ of the assembling filaments as a function of time and salt concentration $\left(c_{S}\right)$ for the given protein concentration of 0.07 $\mathrm{g} / \mathrm{L}$. We show that filament formation is accelerated with increasing $c_{S}$. At low $c_{S}(10 \mathrm{mM} \mathrm{KCl})$ no lateral or elongational growth is observed, whereas at $c_{S}=50-200 \mathrm{mM}$, the hydrodynamic cross-sectional radius and the elongation rate increases with $c_{S}$. $R_{g}$ versus $M_{w}$ plots suggest that the mass per unit length increases with increasing salt content, which is confirmed by STEM mass measurements. A kinetic model based on rate equations for a two step process is able to accurately describe the variation of mass, length and diffusion coefficient of the filaments with time and provides a consistent description of the elongation accelerated by increasing $c_{S}$.

\section{Introduction}

The cytoskeleton is a composite network of three distinct filament networks along with motor proteins and cross linkers ${ }^{1}$. Intermediate filaments (IFs) constitute one of the filament networks and are expressed in a cell-type specific manner. Despite their important role in cell mechanics ${ }^{115}$ they are the least well understood among the cytoskeletal filaments. Remarkably, they are more flexible than actin filaments and microtubules $\frac{6.8}{6}$, display high tensile strength 910 and striking extensibility up to a strain of at least $3.5 \frac{10111}{1}$. A likely hypothesis is that these intriguing mechanical properties are defined by their molecular

\footnotetext{
${ }^{a}$ Chemistry Department, University of Paderborn, 33098 Paderborn, Germany E-mail: klaus.huber@upb.de

${ }^{b}$ Institute for X-Ray Physics, University of Goettingen, 37077 Goettingen, Germany. E-mail: sarah.koester@phys.uni-goettingen.de

c Biological Soft Matter Group, AMOLF, Science Park 102, Amsterdam, NL-1098XG, The Netherlands.

$\dagger$ Electronic Supplementary Information (ESI) available: [details of any supplementary information available should be included here]. See DOI: 10.1039/b000000x/ $\ddagger$ Current address: Institute of Physical Chemistry, RWTH Aachen, D-52056 Aachen, Germany
}

architecture. Thus, a thorough understanding of the assembly pathway, kinetics, and dependence on protein concentration and solution conditions is indispensable for eventually unraveling cell mechanics.

Vimentin is the IF protein typically found in the cytoskeleton of mesenchymal cells $\frac{1213}{13}$. Under denaturing conditions, purified vimentin exists as monomers of molecular weight $M_{w}=5.4 \times 10^{4}$ $\mathrm{g} / \mathrm{mol}$ and forms first parallel dimers and then antiparallel, half-staggered tetramers of $M_{w} \simeq 2.15 \times 10^{5} \mathrm{~g} / \mathrm{mol}$ in physiological, but low salt buffers. Upon addition of monovalent salt such as $\mathrm{KCl}$ in a physiological concentration range, typically 8 tetramers assemble laterally into a unit-length filament (ULF) over a timescale of seconds to minutes $14-20$. In a second step these ULFs elongate via a step growth polymerization based on an end-to-end annealing of ULFs and filaments over timescales of minutes to hours $1421+23$. The lateral assembly step can be followed by small angle x-ray scattering (SAXS) 24|26]. For the elongation step, most previous mechanistic studies have relied on electron microscopy 18212728 , which provides excellent spatial resolution, but has a limited time resolution and may be highly invasive concerning sample preparation. It should be noted, 
however, that the above described pathway has been derived for a simplified in vitro system similar to what we are studying in our present work. In the physiological environment of a cell, additional mechanisms may play a role, such as binding proteins, enzymes or post-translational modifications. Due to the high complexity in the cell, bottom-up approaches employing purified protein systems are important in order to understand the principal underlying mechanisms and interactions. These previous studies have revealed important information: vimentin assembly is sensitive to temperature ${ }^{22}$, salt concentration $17|25| 27$ and mixing conditions 29 . We can safely assume a dependence on protein concentration as well. Moreover, SAXS data indicate that the essential features of the two-step mechanism are insensitive to the salt concentration and the ion type, i.e. $\mathrm{KCl}$ or $\mathrm{MgCl}_{2}$. However, a slight increase of the filament cross section with increasing salt concentration could be resolved ${ }^{17}$, whereby the effect turned out to be more pronounced for magnesium ions than for sodium ions. Unfortunately, SAXS is not capable of following the elongation of filaments starting out with ULFs, since the resulting length values exceed the size scale accessible to SAXS. In order to fill this gap, we applied multi-angle time resolved light scattering in a preceding work and succeeded to characterize the growing vimentin filaments by means of their particle mass and elongation up to a length of half a micron. 14 The evolution of filament mass and length could be successfully reproduced with a kinetic model including the step of ULF formation and of filament elongation. The process was triggered at a $\mathrm{KCl}$ concentration of $c_{S}=50 \mathrm{mM}$. It is still unknown, how a variation in the content of $\mathrm{KCl}$ affects the elongation process and how the kinetic model parameters would account for such a variation.

Here we investigate the effect of varying the concentration of $\mathrm{KCl}$ as an added monovalent salt on the structure and elongation kinetics of vimentin filaments. The following questions shall be answered: To which extent does the addition of $\mathrm{KCl}$ influence filament formation and elongation? If trends can be identified, will the kinetic model introduced in ref 14 be able to interpret these trends with physically meaningful kinetic parameters? Does a variation in the concentration of the added salt also have an effect on the nature of the filament cross section? In order to tackle these problems, we combine time-resolved static and dynamic light scattering (SLS, DLS) as in one of our earlier studies ${ }^{14}$ with direct imaging by scanning electron microscopy (STEM) and we extend the concentration regime of added $\mathrm{KCl}$ from $100 \mathrm{mM}$ analysed thus far $\frac{17}{17}$ to $200 \mathrm{mM}$. While SLS and DLS allow us to follow the filament elongation and to simultaneously record the mass, size and diffusion coefficient of the assembling species, STEM provides a direct, quantitative measure of the filaments formed. STEM has proven to be a powerful technique to directly determine the protein mass while obtaining high resolution images of their structure $15 \mid 30$. By measuring the number of scattered electrons in a STEM image, which is proportional to the particle mass, it is possible to perform in situ mass determination of unstained biological structures, regardless of their shape. We confirm the validity of the two step process and quantitatively model the growth kinetics and thereby reveal further insight into how the filaments connect end-to-end. Additionally, we reveal an influence of the added $\mathrm{KCl}$ on the cross section of the elongating filaments.

\section{Methods}

Sample preparation and the instrument setup for light scattering have been described in ref 14 . In brief, human vimentin protein was recombinantly expressed in Escherichia coli, purified and stored in $8 \mathrm{M}$ Urea, $5 \mathrm{mM}$ Tris-hydrochloric acid ( $\mathrm{pH}=7.5$ ), $1 \mathrm{mM}$ ethylene glycol tetraacetic acid, $0.1 \mathrm{mM}$ ethylene glycol tetraacetic acid, $1 \mathrm{mM}$ dithiothreitol, and $10 \mathrm{mM}$ methyl ammonium chloride at $-80^{\circ} \mathrm{C}$. Before experiments, the solutions were stepwise dialyzed into $2 \mathrm{mM}$ sodium phosphate buffer. Cylindrical quartz cuvettes with a diameter of $20 \mathrm{~mm}$ were used as scattering cells. $3 \mathrm{~mL}$ of vimentin solution were filtered into cells using $0.22 \mu \mathrm{m}$ cellulose acetate filters (Chromafil, Macherey-Nagel, Düren, Germany) to remove dust. Assembly was triggered by addition of $3 \mathrm{~mL}$ of filtered $\mathrm{KCl}$ solution in phosphate buffer at twice the required final concentration of $\mathrm{KCl}$, followed by gentle shaking to promote mixing. The final protein concentration was kept constant at $0.07 \mathrm{~g} / \mathrm{L}(0.33 \mu \mathrm{M}$ of tetramers $)$ and the final $\mathrm{KCl}$ concentration was varied between $200 \mathrm{mM}$ and $10 \mathrm{mM}$. Time resolved SLS and DLS measurements were performed on a commercial setup (ALV-CGS-3, ALV GmbH, Langen, Germany), with 8 detectors spaced at angular intervals of $8^{\circ}$ covering a $q$ range of $0.0046 \leq q \leq 0.017 \mathrm{~nm}^{-1}$, where $q=4 \pi n \sin (\theta / 2) / \lambda$ is the scattering wavevector, where $n$ is the refractive index of the solvent, $\theta$ is the scattering angle and $\lambda$ is the wavelength of the incident light. For single angle readings, an ALV-CGS-5000E (ALV GmbH, Langen, Germany) combined SLS/DLS instrument was used, covering a $q$ range of $0.0046 \leq q \leq 0.024 \mathrm{~nm}^{-1}$, which corresponds to dimensions of $200-50 \mathrm{~nm}$.

For scanning transmission electron microscopy, vimentin was assembled under identical protein concentrations and salt conditions as those used for light scattering studies. Imaging was performed on High-Angle Annular Dark Field Mode (HAADF) using a Verios 460 electron microscope (FEI, Hillsboro, OR, USA) operating at an acceleration voltage of $20 \mathrm{kV}$. Absolute molecular mass determination ("mass mapping") was achieved by calibrating the collected electron beam signal using Tobacco Mosaic Virus (TMV) as an internal mass calibration standard. TMV $(0.025 \mathrm{~g} / \mathrm{L}$ in phosphate buffered saline) was added to the carbon-coated copper grids (Ted Pella, Redding, CA, USA) prior to use, rinsed 3 times with Milli-Q water and blotted dry. Following assembly of vimentin for 15 minutes, the protein solution was diluted by a factor of two with assembly buffer and immediately deposited on the grids. After 1 minute of deposition, the grids were rinsed with assembly buffer and adsorbed filaments were fixated by incubating with $0.1 \%$ glutaraldehyde dissolved in Milli-Q water Merck KGaA, Darmstadt, Germany) for 5 minutes. Before imaging, grids were rinsed 5 times with Milli-Q water and air-dried.

Depending on the image contrast between the filaments and the background, HAADF images were analyzed with either an automated custom Python (Python Software Foundation, Beaverton, OR, USA) script (100 - $200 \mathrm{mM}$ salt conditions) or with a manual 
bounding box approach using ImageJ (NIH, Bethesda, Maryland, $\mathrm{MD}$, U.S.), as described elsewhere ${ }^{31}$ (50 mM salt). In the custom script, the filament borders were automatically detected using a modified Canny edge detector based on the 2D wavelet transform 32 . To measure the distribution of mass per length, the image intensity was measured at consecutive points along the filament axis. The $M_{L}$ at each point was then computed by integrating the measured intensity across the detected filament width. The values were binned along a section of a filament and a batch of such binned values was collected from 50-100 filaments. Finally, histograms were generated from each batch of mass per unit length values.

\section{Data Evaluation}

The variation of the SLS signal with the observation angle and solute concentration can be approximated via Zimm's formula 33 34:

$$
\frac{K c}{\Delta R_{\theta}}=\frac{1}{M_{w}}\left(1+\frac{q^{2}\left\langle R_{g}^{2}\right\rangle_{z}}{3}\right)+2 A_{2} c
$$

where $\Delta R_{\theta}$ is the excess scattering signal recorded at the observation angle $\theta$ from the solute expressed in terms of the absolute scattering cross section per unit volume, $c$ is the concentration of the solute in $\mathrm{g} / \mathrm{L}, K$ is a constant describing the scattering contrast of the solute $\left(2.44 \times 10^{-7} \mathrm{~cm}^{2} \mathrm{~g}^{-2} \mathrm{~mol}^{-1}\right), q$ is the magnitude of the scattering vector, $M_{w}$ is the weight averaged molecular weight of the solute, $\left\langle R_{g}^{2}\right\rangle_{z}$ is the $z$-averaged squared radius of gyration and $A_{2}$ the second virial coefficient. The Zimm formula ${ }^{33} 34$ is valid for $q R_{g} \lesssim 1$, where $R_{g}=\sqrt{\left\langle R_{g}^{2}\right\rangle_{z}}$. SLS data were fitted according to eq 1 assuming $A_{2}=0$. Calibration to absolute units was carried out against toluene.

The primary result of DLS is the field autocorrelation function of the scattered light, which can be fitted to a modified cumulant expansion 35 . 37 :

$$
g_{1}(t, q)=e^{-\mu_{1} t}\left(1+\frac{\mu_{2} t^{2}}{2}\right)
$$

where $\mu_{1}$ and $\mu_{2}$ are the first and second cumulants, respectively. $\mu_{1}$ in the low $q$ limit varies as

$$
\mu_{1} / q^{2}=D_{z}\left(1+q^{2}\left\langle R_{g}^{2}\right\rangle_{z} C+k_{D} c\right) .
$$

$C$ is a constant dependent on particle shape and polydispersity $1438, D_{z}$ is the $\mathrm{z}$-averaged diffusion coefficient (see Supporting Information, eq S3) and $k_{D}$ accounts for the concentration dependence of $\mu_{1}$. DLS data were fitted to a modified cumulant expansion with eq 2 as detailed in an earlier publication 14 . The resulting diffusion coefficients were fitted with eq 3 assuming $k_{D}$ $=0$. The Stokes-Einstein equation relates $D_{z}$ to $R_{H}$ :

$$
R_{H}=\frac{k_{B} T}{6 \pi \eta D_{z}}
$$

In contrast to $R_{g}$, which is a geometric quantity, $R_{H}$ is the radius of a sphere with the same diffusion coefficient as the one of the particle under consideration. The ratio $\rho=R_{g} / R_{H}$ is shape dependent and varies from $\rho \simeq 0.8$ for spheres to $\rho \simeq 2$ for polydisperse rods 3839 . All data were evaluated according to eqs $1-4$ in the $q R_{g} \lesssim 1$ regime.

\section{Results and discussion}

\subsection{General features of vimentin assembly}

Measurements on the starting solutions of purified vimentin yield $M_{w} \simeq 3.3 \times 10^{5} \mathrm{~g} / \mathrm{mol}, R_{g} \simeq 25 \mathrm{~nm}$ and $R_{H} \simeq 15 \mathrm{~nm}$ for all samples. These values are larger than those of a tetramer, corresponding to $M_{w}=2.1 \times 10^{5} \mathrm{~g} / \mathrm{mol}, R_{g} \simeq 17 \mathrm{~nm}$. Following our earlier work ${ }^{14}$, we assume a bidiperse population, consisting of tetramers $(\simeq 95 \%)$ and dimeric ULFs, assembled end-to-end $(\simeq$ $1-5 \mathrm{wt} \%)$. Alternatively, premature assembly or aggregation of vimentin subunits may be the cause for the increased value of $R_{g}$. However, the choice of initial conditions does not significantly affect the modeling presented further below.

Assembly of vimentin was initiated by addition of $3 \mathrm{~mL}$ of $\mathrm{KCl}$ solution to $3 \mathrm{~mL}$ of the vimentin solution. The kinetic run for $c_{S}=$ $50 \mathrm{mM} \mathrm{KCl}$ was already reported in an earlier publication $\frac{14}{14}$ and is included here to complement the range of salt concentrations. The salt and protein concentrations quoted in this manuscript refer to the final assembly concentration of the solutions after mixing. Note that for the STEM experiments we additionally dilute the solution prior to imaging. The vimentin concentration was $0.07 \mathrm{~g} / \mathrm{L}$ for all samples and the $\mathrm{KCl}$ concentrations were 10, 50, 100 and $200 \mathrm{M}$.

Figure 1 shows $M_{w}, R_{g}, R_{H}$ and $\rho=R_{g} / R_{H}$ as a function of time

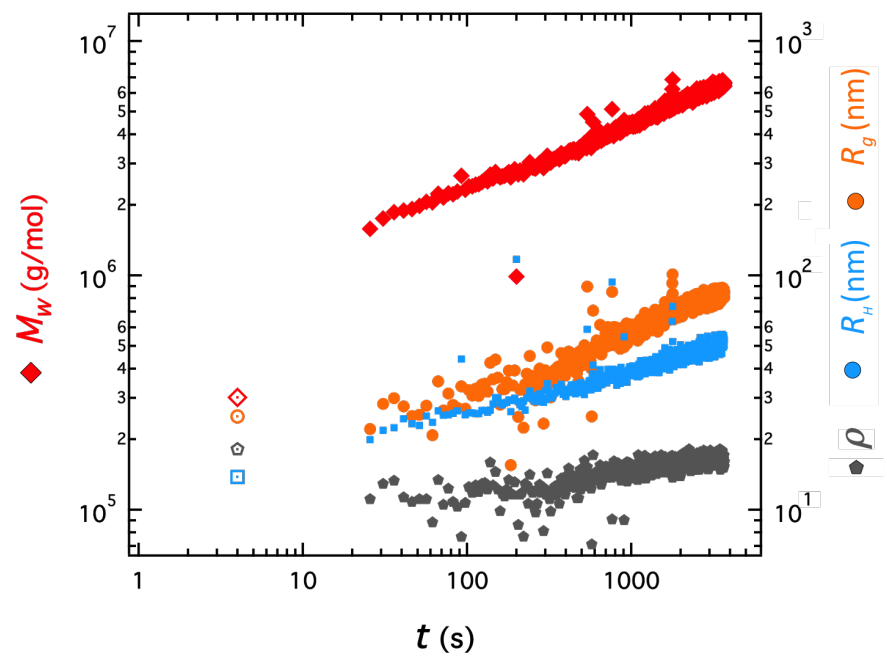

Fig. 1 Vimentin assembly kinetics as revealed by TR-SLS/DLS. $M_{w}, R_{g}$, $R_{H}$ and $\rho$ as a function of time for a vimentin solution with $c_{p}=0.07 \mathrm{~g} / \mathrm{L}$ and $c_{S}=100 \mathrm{mM}$. $\rho$ is multiplied by a factor of 10 for better visibility. Symbols located at $t=3 \mathrm{~s}$ are the values measured prior to the addition of $\mathrm{KCl}$. The data reveal a fast increase in molar mass at approximately constant radius of gyration for time points $t \lesssim 50 \mathrm{~s}$, followed by a steady increase in both mass and size at longer times.

for vimentin with $100 \mathrm{mM} \mathrm{KCl}$, as a representative example. The data are consistent with the two step model proposed by Hermann et al. 1516 The pronounced increase in $M_{w}(\simeq \times 6)$ compared to the initial state without an increase in $R_{g}$ at early times, along with a decrease in $\rho$ is likely caused by lateral assembly of the tetramers into ULFs. For $t \geq 100 \mathrm{~s}$, the increase of $M_{w}, R_{g}, R_{H}$ 
and $\rho$ indicates longitudinal growth.

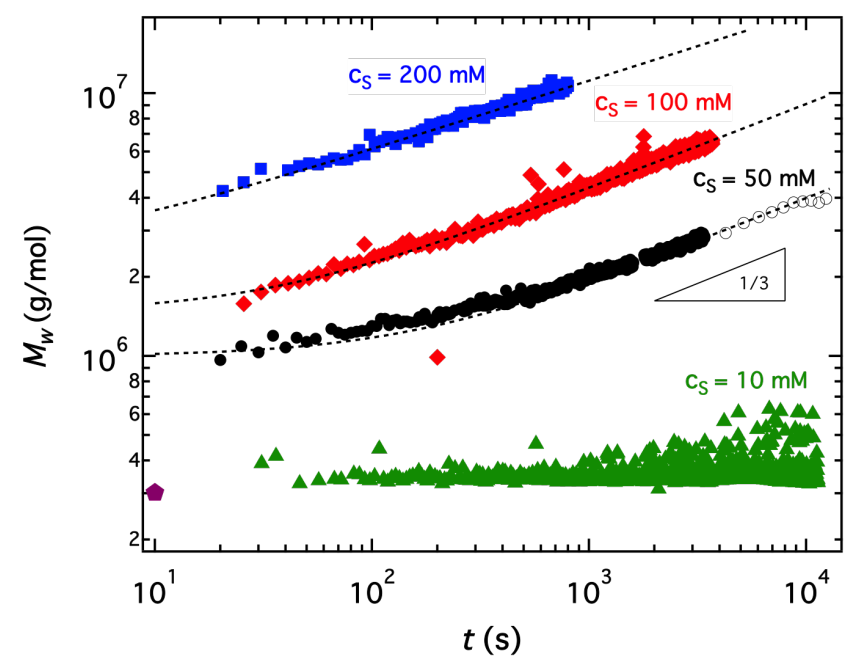

Fig. $2 M_{w}$ plotted against time for samples with $c_{p}=0.07 \mathrm{~g} / \mathrm{L}$ and $c_{S}=$ $10 \mathrm{mM}$ (green triangles), $50 \mathrm{mM}$ (black circles), $100 \mathrm{mM}$ (red diamonds) and $200 \mathrm{mM}$ (blue squares). The purple pentagon at $t=10 \mathrm{~s}$ is the value of $M_{w}$ prior to the addition of $\mathrm{KCl}$. Black hollow circles correspond to 30 angle sweeps covering a range of $0.0046 \leq q \leq 0.024 \mathrm{~nm}^{-1}$. Dashed lines are fits to the scaling model (eq 8 .

To test the influence of salt concentration on the assembly conditions, we next compared the time dependence of $M_{w}$ for $\mathrm{KCl}$ concentrations ranging from 10 to $200 \mathrm{mM}$ (Figure 2). For $c_{S}=$ $10 \mathrm{mM}$, no measurable assembly takes place over one hour. This contrasts with previous reports which found assembly starting at $10 \mathrm{mM} \mathrm{KCl}$, albeit at much higher protein concentration in the range of $0.5-5 \mathrm{~g} / \mathrm{L}^{1726}$, and indicates a protein concentration dependence of the assembly. For $c_{S}=50-200 \mathrm{mM}$, increasing $c_{S}$ leads to faster assembly kinetics, that is, at any given time, the $M_{w}$ of the filaments is larger for higher $c_{S}$. Qualitatively, the mechanism appears to be the same, with all samples displaying a power law behavior of $M_{w} \propto t^{\zeta}$ with $\zeta \simeq 1 / 3$ in the long time limit. At long times for the $c_{S}=50 \mathrm{mM}$ dataset, $M_{w}$ decreases, presumably due to sedimentation 14 , we therefore consider only the first six hollow datapoints in the following plots.

In the following, we outline a scaling model for the kinetics of filament elongation to show that such exponents are consistent with rods annealing end-to-end.

In the case of instantaneous lateral association, the concentration of filaments $\left[F_{i}\right]$ with degree of polymerization $i$ corresponding to the number of ULFs, is given by:

$$
\frac{d\left[F_{i}\right]}{d t}=-\sum_{j=1}^{\infty} k_{i, j}\left[F_{i}\right]\left[F_{j}\right]+\frac{1}{2} \sum_{j=1}^{i-1} k_{j, i-j}\left[F_{j}\right]\left[F_{i-j}\right]
$$

The first term describes the loss of filaments of longitudinal degree of polymerization $i$ by reaction with other filaments of any size. The second term accounts for the generation of filaments of degree of polymerization $i$ by the reaction of filaments with degree of polymerisation $j$ and $i-j$. The reaction constant for filaments with longitudinal degree of polymerization $i$ and $j, k_{i, j}$ is given by:

$$
k_{i, j} \sim\left(D_{i}+D_{j}\right)\left(R_{i}+R_{j}\right) P_{i, j}
$$

In eq $6 R_{i}$ is the size of particle $i, D_{i}$ is the corresponding diffusion coefficient, and $P_{i, j}$ is the probability that a collision results in annealing. Following the presentation by Carrotta et al. $\frac{40}{4 i, j}$ scales as:

$$
k_{a i, a j}=a^{\varepsilon} k_{i, j}
$$

where $\varepsilon$ is a constant that sets the scaling of $k_{i, j}$ with filament longitudinal degree of polymerisation $a \times i$. An approximate solution for the weight averaged molar mass of the filament ensemble is given by:

$$
M_{w} \simeq M_{0}[1+(1-\varepsilon) \kappa t]^{1 /(1-\varepsilon)}
$$

where $M_{0}$ is the mass of a monomer and $\kappa$ is a reaction constant proportional to $k_{1,1}$.

The dependence of $k_{i, j}$ on length arises largely due to $P_{i, j}$, which varies as $P_{i, j} \propto(i+j)^{-\beta}$. If the $R$ and $D$ dependences in eq 6 cancel, then $\varepsilon=-\beta$, see ref 40 . A value of $\beta=2$ was derived by Hill for rods assuming the product of the maximum angle and maximum distance between two rods required for a successful collision are independent of rod length 41 , which leads to a predicted dependence of $M_{w} \propto t^{1 / 3}$ at long times, in reasonable agreement with our experimental observations. Fits to eq 8 are presented in Figure 2 with the resulting fit parameters given in Table 1. The scaling model correctly describes the experimental data. The trends observed in the reaction constants parallel those obtained from fitting the more complete kinetic model, which is discussed further below.

Figure 3 shows $R_{g}, R_{H}$ and $\rho$ as a function of $M_{w}$ for kinetic runs with $c_{S}=50,100$ and $200 \mathrm{mM}$. In general, the following scaling laws apply to colloids with fractal dimension $1 / \alpha$ :

$$
R_{g} \simeq R \sim M^{\alpha} ; \quad D \sim 1 / R_{H} \sim M^{-\alpha}
$$

The relationships between the measured parameters are summarized in Table 1. In the limit of self-similar morphological transformations, an exponent of $\alpha=1$ is expected for eq 9 for infinitely thin rods. The exponents for $R_{g}$ are slightly larger than 1 but closer to the expected value than for $R_{H}$, which reveals smaller values than expected. Two causes for deviations from the expected scaling laws can be identified, a non-negligible cross section whose impact on $R_{H}$ decreases with increasing length and a varying polydispersity in the length distribution. Apparently the impact of a finite cross section (with diameter $d$ ) on $R_{g}$ ceases already at lower filament length than the impact of the hydrodynamically effective cross section (with diameter $d_{H}$ ) does on $R_{H}$. This is in agreement with theoretical relations for cylinders: $R_{g} \simeq \sqrt{L^{2} / 12+d^{2} / 8}$ and $R_{H} \simeq L / \ln \left(L / d_{H}\right)$. The increasing effect of $d_{H}$ with decreasing $L$ may easily cause an increase in $R_{H}$ at a roughly constant $R_{g}$, hence leading to lower apparent exponents of $\alpha$ for $R_{H}$ (see Table 1 . The values of $\alpha>1$ for the $R_{g}$ datasets may be attributed to a polydispersity, which slightly increases as the assembly progresses. $\frac{14}{14}$ The parameter $\rho$ exhibits the same increase from values close to 1 to values close to 2 for the three runs considered. Whereas a value of 1 is compatible with the shape of 


\begin{tabular}{c|cc|cc|ccc} 
& \multicolumn{2}{c}{$R_{g}=B M_{w}^{\alpha}$} & \multicolumn{2}{c}{$R_{H}=C M_{w}^{\alpha}$} & \multicolumn{3}{c}{ Scaling model } \\
$c_{S}(\mathrm{mM})$ & $B$ & $\alpha$ & $C$ & $\alpha$ & $p$ & $\varepsilon$ & $\kappa\left(\mathrm{s}^{-1}\right)$ \\
\hline 200 & $2.8 \times 10^{-8}$ & 1.35 & 0.000052 & 0.86 & 12 & -2.8 & 0.078 \\
\hline 100 & $6.0 \times 10^{-8}$ & 1.05 & 0.0019 & 0.65 & 6.8 & -2.1 & 0.0093 \\
\hline 50 & $4.5 \times 10^{-6}$ & 1.25 & 0.0042 & 0.605 & 4.6 & -2 & 0.0021
\end{tabular}

Table 1 Apparent power laws describing the variation of $R_{g}$ and $R_{H}$ with $M_{w}$ for different $\mathrm{KCl}$ concentrations. $M_{w}$ is in $\mathrm{g} / \mathrm{mol}$ and $R_{g}$ and $R_{H}$ in $\mathrm{nm}$. Scaling model: Kinetic parameters obtained from eqs $7,8 p$ is obtained from fits to $R_{g}=M_{w} /\left(\sqrt{12} p M_{L, t e t}\right)$, where $M_{L, t e t}=3570 \mathrm{gmol}^{-1} \mathrm{~nm}^{-1}$ is the mass per unit length of a tetramer. See Figure 3 for plots of $R_{g}$ and $R_{H}$ against $M_{w}$, from which $p$ for the scaling model is extracted.
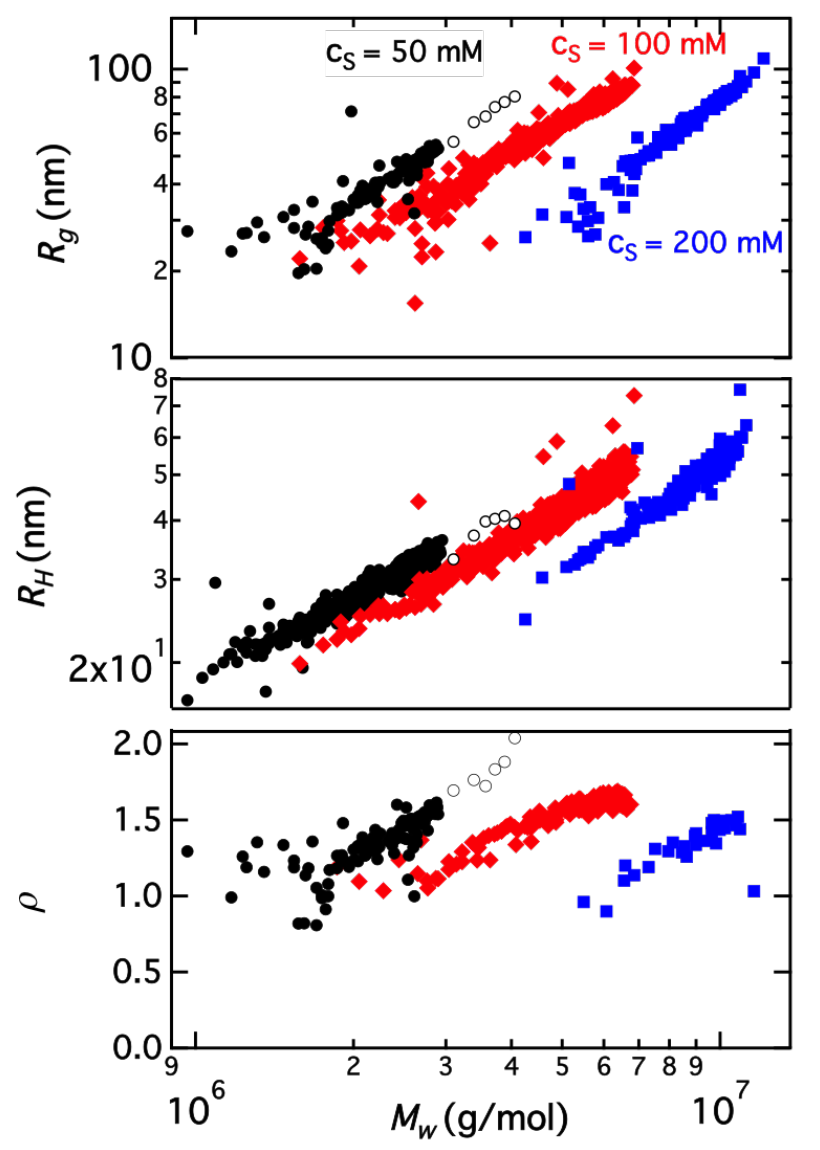

Fig. $3 R_{g}$ versus $M_{w}$ (top), $R_{H}$ (middle) and $\rho=R_{g} / R_{H}$ (bottom) versus $M_{w}$ recorded for $c_{S}=50 \mathrm{mM}$ (black squares), $100 \mathrm{mM}$ (red diamonds) and $200 \mathrm{mM}$ (blue squares). Hollow symbols correspond to 30 angle sweeps. Each five points for the $R_{g}$ versus $M_{w}$ dataset recorded for $c_{S}=50 \mathrm{mM}$ and all datasets for $\rho$ have been binned into a single point for clarity.

a compact, slightly anisotropic ULF, values larger than 1.5 indicate rod-like structures. Hence the variation in $\rho$ nicely supports the same transformation of ULFs to rod-like filaments based on ULFs as constituent units at all three salt concentrations.

One more aspect in Figure 3 which deserves attention is the lateral shifts in the $R_{g}$ versus $M_{w}$ and, in line with this, the $\rho$ versus $M_{w}$ datasets, appearing with increasing salt content $c_{S}$. These offsets may be caused by either greater lateral assembly or by incomplete consumption of tetramers. ${ }^{42}$ A greater lateral assembly would lead to an increased mass per unit length of the elongating filaments and hence could explain in a straightforward man- ner the increase of $M_{w}$ at a given size $R_{g}$ since an increase of the cross section affects $M_{w}$ but hardly changes $R_{g}$. An incomplete consumption of monomers as an alternative explanation for the offsets is based on eq 11, applied to extract values for $M_{w}$. Values for $M_{w}$ resulting from eq 1 in turn rely on concentration values used for $c$. Under the assumption of complete consumption of monomers, $c$ would correspond to the total mass of protein in solution. If, however, a fraction of that protein remained as tetramers in equilibrium, $M_{w}$ would not exclusively refer to the filaments but instead correspond to a weight average of filaments and non-consumed tetramers, with the latter having a significantly lower mass per unit length. It is this discrepancy which would then cause a shift in the plot of $R_{g}$ versus $M_{w}$.

Looking at the data, a first hint for the occurrence of a greater lateral assembly as the cause of the shifts is derived from the SLS/DLS results. If no variation in thickness occurred with increasing salt concentration, a plot of $R_{H}$ versus $R_{g}$ would be expected to reveal correlations which perfectly overlay. If, on the other hand, the cross section increases with concentration for a given filament length, the impact on $R_{H}$ would be larger than on $R_{g}$ as $R_{H}$ is more sensitive to cross-sectional thickness. An increase of the cross section would then impose a shift in a plot of $R_{H}$ versus $R_{g}$. Such a shift is in fact observed in Figure S1 of the Supporting Information, in particular when increasing $c_{S}$ from $100 \mathrm{mM}$ to $200 \mathrm{mM}$, supporting the scenario of greater lateral assembly.

Literature data on this topic are not yet conclusive. Two studies by Herrmannn et al. $\frac{15 / 16}{}$ provide evidence for the existence of entities smaller than ULFs in $100 \mathrm{mM}$ salt solution and thus do not exclude the existence of a non-assembled pool of tetramers at equilibrium. Experiments by Nöding et al. .43 in $100 \mathrm{mM} \mathrm{KCl}$ indicate that, while a finite concentration of soluble tetramers exists, it is rather low and Portet et al. ${ }^{22}$ find full consumption of tetramers at the same $c_{S}$. SAXS experiments by Brennich et al. 17 provide evidence for an increasing cross sectional diameter of vimentin filaments with increasing $c_{S}$ and suggest that a fraction of tetramers remains at low salt concentrations (10 to $20 \mathrm{mM}$ ) but not for $c_{S}>20 \mathrm{mM}$. If full tetramer consumption is assumed for $c_{S}=100 \mathrm{mM}$, the mass per unit length of the filaments calculated from LS data corresponds to $p=7 \pm 1$ tetramers per cross section, in agreement with earlier reports at similar salt concentrations $(p=8)^{21 / 27 / 44}$. This suggests that tetramer consumption is complete or nearly complete for $c_{S}=100 \mathrm{mM}$, and that incomplete tetramer consumption plays a role only at salt concentrations below $100 \mathrm{mM}$ if at all. In order to directly test this hypothesis we carried out STEM experiments, which 
provide a direct measure of the mass per unit length of filaments.

Figure 4 summarizes the STEM results for vimentin selfassembly at different $\mathrm{KCl}$ concentrations (50, 100 and $200 \mathrm{mM}$ ). More STEM images are provided in the supporting information, see Figure S4. No assembly was observable at $c_{S}=10 \mathrm{mM}$, in agreement with the light scattering results. At $c_{S}=50 \mathrm{mM}$ the filament intensity was observed to be too low for accurate determination by automated image detection and instead a bounding box algorithm ${ }^{31}$ was applied, which accurately determines the filament $M_{L}$ but is not able to determine filament width. In the $c_{S}$ range of 50-200 mM, the mass per unit length $\left(M_{L}\right)$ and filament widths $(d)$ show considerable polydispersity. Histograms were fitted to a Gaussian function: $f(x)=A \exp \left[-(x-\bar{x})^{2} /\left(2 \sigma^{2}\right)\right]$, where $\sigma$ is a measure of the spread of the distribution and $\bar{x}$ corresponds to the position of the peak, and thus the average value and the variance of the distributions for a given $c_{S}$ are extracted. The parameter $\sigma$ serves as a measure of the spread of $M_{L}$ and $d$ values for a given salt concentration. $M_{L}$ for $c_{S}=200 \mathrm{mM}$ is noticeably higher than for $c_{S}=50$ and $100 \mathrm{mM}$. Multiple images, comprising a total of between 50-100 individual filaments were analysed for each salt concentration using the aforementioned procedure. The error bars for the average $M_{L}$ and for $\sigma$ were calculated as twice the standard deviation ( $\simeq 95 \%$ confidence intervals) of the different batches analyzed for a given salt concentration. This increase in $M_{L}$ suggests that the shifts observed in Figure 3, at least in case of $c_{S}=200 \mathrm{mM}$, correspond to greater lateral assembly of the tetramers and not to incomplete tetramer consumption. The shift between concentrations $c_{S}=50 \mathrm{mM}$ and $c_{S}=100 \mathrm{mM}$ may result from incomplete tetramer consumption. It is not possible to clearly resolve this feature at this stage. We discuss the trends for these two quantities with salt concentration in more detail in the next two sections.

\subsection{Application of the kinetic model}

In order to quantify the conclusions presented above, we apply the two step kinetic model. The first step consists of lateral assembly of tetramers with rate constant $k_{n}$, and the second of end-toend annealing of these ULFs. A derivation of the kinetic model has been presented in an earlier publication ${ }^{14}$ and is also included in the Supporting Information.

Figure 5 shows $M_{w}, R_{g}$ and $R_{H}$ as a function of time for $c_{S}=$ 50,100 and $200 \mathrm{mM}$ along with the fits to the two step kinetic model (eqs S18-S20). The fits describe the data well with five free parameters: $k_{n}, p, A, \beta$ and $d_{H}$, where $\beta$ and $A$ (with units of $\mathrm{nm}^{\beta}$ ) determine the rate constant of elongation, as discussed below and in the Supporting Information. Noticeable deviations between experiment and model occur only for the $R_{H}$ datasets, in particular for $c_{S}=200 \mathrm{mM}$. We show in the Supporting Information that the faster increase of data compared to the model can be assigned to $\mathrm{a} \pm 20 \%$ change in the cross sectional hydrodynamic diameter. Kinetic modeling revealed some trends of the fitted parameters, which are summarized in Table 2 and which shall be discussed in the following in more detail.

The kinetic runs for $c_{S}=100 \mathrm{mM}$ and $200 \mathrm{mM}$ are well modeled assuming that lateral assembly occurs instantaneously $\left(k_{n}=\infty\right)$, while for $c_{S}=50 \mathrm{mM}$, a finite value of $k_{n}$ is used as described in our earlier work 14 .

The exponent $\beta$ is close to 4 at all three salt concentrations and thus turned out to be insensitive to the variation of $c_{S}$. The exponent $\beta$ was therefore fixed at the value of 4 , and used in a second fit resulting in the values for the model parameters $k_{n}$, $A$ and $d_{H}$ given in Table 2.. This differs from Hill's calculation of $\beta \simeq 2$, which gives a poorer fit to the results, particularly the $M_{w}$ versus time dataset, see ref 14 . The value of $\beta \simeq 4$ may be interpreted as a stronger length dependence of the rate constant $k_{i, j}$ for filament elongation than predicted by Hill's theory 41 . We note that while $\beta \simeq 4$ gives a significantly better fit than Hill's value, this may be an artifact related to the use of an average reaction constant instead of fully accounting for the distribution of filament lengths (see Supporting Information).

The most striking trend is observed with the parameter $A$, which reflects the increase in assembly speed of the filaments with $c_{S}$. The kinetic model responds to this increase in speed with an increase of the rate constant for elongation, which is accomplished by the parameter $A$ in eq S11. The parameter $A$ has a simple geometrical interpretation when $\beta=4$ : If a sphere with diameter $L$ is drawn around the reacting rods in the second step, and two areas with a maximum value of $\sqrt{A}$ are drawn at opposite ends of each sphere, a collision between rods is successful when the drawn areas of the spheres touch, see Supporting Information Figure S3. Hill's calculation of $\beta=2$ results from assuming that a collision between two filaments leads to elongation if the maximum distance and maximum angle between the filaments are below a constant value $14 / 41$.

Finally, $d_{H}$ increases with $c_{S}$ with a particularly strong increment between $100 \mathrm{mM}$ and $200 \mathrm{mM}$. A small increase in the cross sectional diameter of mature filaments with increasing $c_{S}$ in the range of $20-100 \mathrm{mM}$ has been reported by Brennich et al. 25 , however, the increase in $d_{H}$ found here is more pronounced.

To summarize, the values obtained from the kinetic model, collected in Table 2, quantify our conclusions that $p, A$ and $d_{H}$ increase with increasing $c_{S}$. The increase in $p$ and $d_{H}$ corresponds to filaments with a higher mass per unit length, and the increase in $A$ to a faster elongation reaction.

\subsection{Structural and hydrodynamic parameters of vimentin fil- aments}

Figure 6 compares the mass per unit length obtained from LS and STEM as a function of salt concentration. Both techniques agree reasonably well within experimental error. Note that the error for LS values arises predominantly from the uncertainty in the concentration of protein in solution. Because the samples were prepared from the same stock solution, this error will affect all three samples by the exact same factor. Hence, the increase in $M_{L}$ between $c_{S}=50-100 \mathrm{mM}\left(M_{L} \simeq 3.5 \times 10^{4} \mathrm{gmol}^{-1} \mathrm{~nm}^{-1}\right)$ and $c_{S}=200 \mathrm{mM}\left(M_{L} \simeq 6 \times 10^{4} \mathrm{gmol}^{-1} \mathrm{~nm}^{-1}\right)$ measured by LS is beyond experimental error. For $c_{S}=50 \mathrm{mM}$ and $c_{S}=100$ $\mathrm{mM}$, our results are consistent with the literature value of $p=8$ observed in the $c_{S}=20-100 \mathrm{mM}$ range $2127 / 44$. At $c_{S}=200$ 

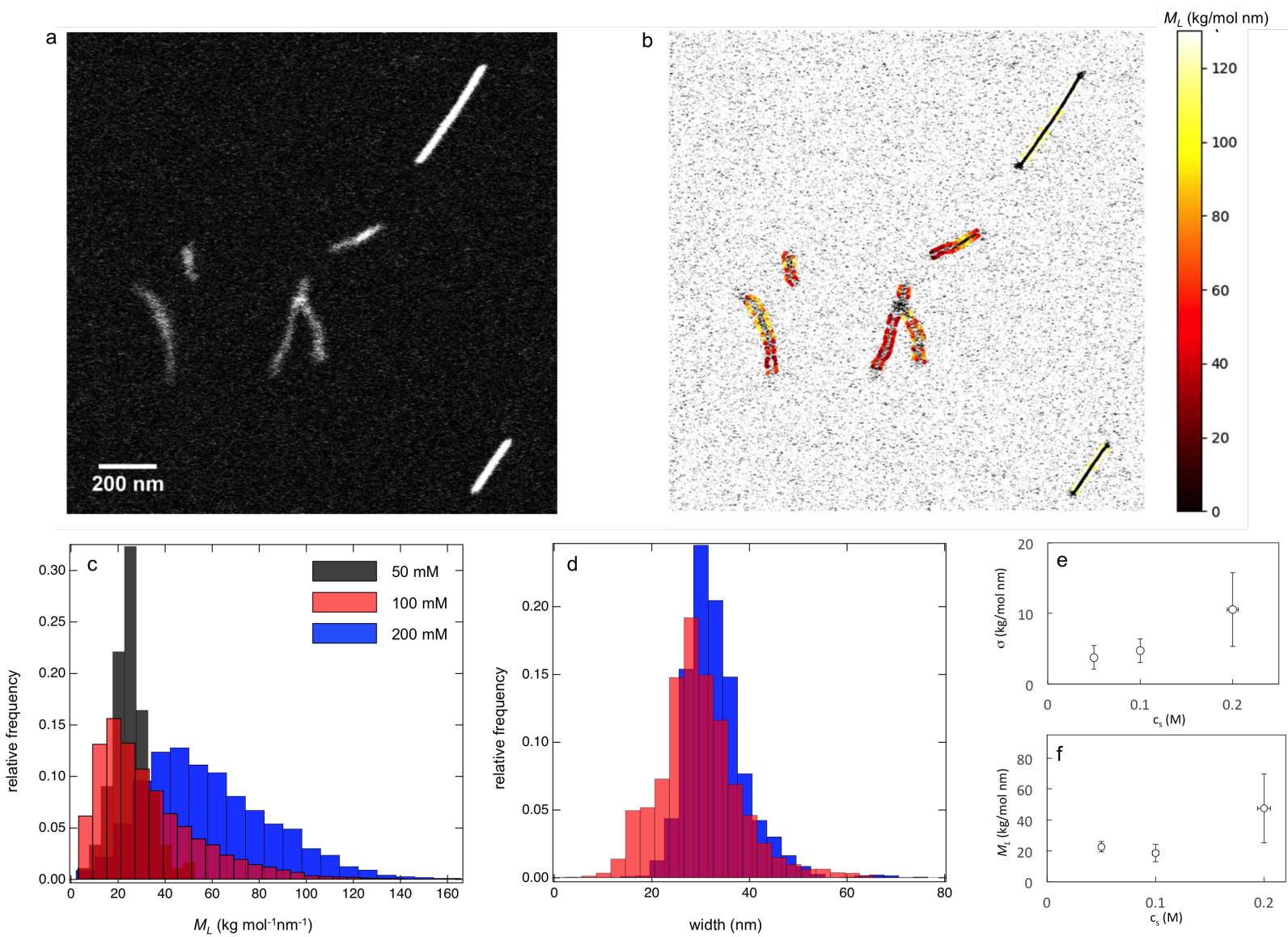

Fig. 4 Summary of STEM results. (a) Example image showing vimentin filaments assembled at $c_{S}=200 \mathrm{mM}$. Image contrast is optimised to aid clarity. TMV (bright rods) are used as internal mass calibration standards. (b) Mass per length $\left(M_{L}\right)$ is extracted from the images using automated image detection. Detected borders are color-coded with the $M_{L}$ at a given position on the filament axis. Histograms show the relative frequency of filament $M_{L}$ (c) and filament width (d) for different salt concentrations. Average values of $M_{L}$ (e) and the spread of the distribution (f) are plotted with respect to salt concentration.

$\mathrm{mM}$, a higher value of the mass per unit length, corresponding to $p \simeq 12$ is observed, in good agreement with earlier reports 15 . Interestingly, the largest jump in mass per unit length occurs between 100 and $200 \mathrm{mM}$, around the average salt concentration in the cytosol of mammalian cells $\left(\simeq 154 \mathrm{mM}^{45}\right)$.

STEM data reveal an increase in the polydispersity in $M_{L}$ with increasing salt concentration. The variation of $M_{L}$ occurs both between filaments and within single filaments. This spread in $M_{L}$ is known to occur when assembly is triggered by a sudden change in the ionic strength of the solution, also called rapid dilution or 'kick-start assembly'15. By contrast, when tetramers are dialyzed against a salt solution, the resulting filaments are less polymorphic. Here, we employ the rapid dilution method because it allows access to the assembly kinetics of vimentin.

Unlike to the trend of $M_{L}$, the diameter of the filaments measured by STEM does not vary between $c_{S}=100$ and $200 \mathrm{mM}$, with a value $d \simeq 29 \mathrm{~nm}$. This is higher than is generally reported for vimentin filaments which have a typical diameter of around 11 $\mathrm{nm}$. We ascribe this discrepancy to flattening of the filaments as they adsorb to the surface, a phenomenon that has previously been reported for soft proteinaceous fibrils in electron microscopy studies 15 . We found that flattening was less pronounced for filaments that were fixated with glutaraldehdye in solution rather than on the grid surface, but unfortunately fixation induced filament bundling, resulting in thicker filaments with greatly increased $M_{L}$. Therefore to ensure accurate $M_{L}$ we performed fixation to the grid surface, with the disadvantage that the filaments appear broader.

The values of $d_{H}$, obtained from fitting the kinetic model to light scattering data show a continuous increase with increasing salt concentration. Whereas $d$ represents a direct measure of the filament width, $d_{H}$ is a hydrodynamic quantity which incorporates effects such as hydration layers as well as ionic friction arising from dissociated charges along the filaments. It is therefore not surprising that the two trends observed do not entirely agree. An increase of $M_{L}$ at constant width, as suggested by the STEM data, implies that filaments become more densely packed as the salt concentration increases.

\section{Conclusions}

The present work studies the impact of added salt concentration on the morphology and assembly kinetics of vimentin filaments. 
Table 2 Kinetic model: Parameters obtained from the two step model fits. Whereas $\beta$ refers to the exponent that gives a best fit to the data, the model parameters $k_{n}, A$ and $d_{H}$ were established with a second fit based on a fixed value of $\beta=4$. Model parameters $\left(k_{n}, A\right.$ and $\left.d_{H}\right)$ established with variable $\beta$ are not shown. The mass per unit length of the filaments is calculated as $M_{L}=60 / 43 p M_{L, t e t}$, where $M_{L, t e t} \simeq 3570 \mathrm{gmol}^{-1} \mathrm{~nm}^{-1}$. The factor of $60 / 43$ arises due to the partial overlap of filaments during the annealing phase.

\begin{tabular}{l|lll|lll}
\multicolumn{4}{c}{ Kinetic } & \multicolumn{4}{c}{ Structural } \\
$c_{S}(\mathrm{mM})$ & $k_{n}(\mathrm{M})$ & $\mathrm{A}\left(\mathrm{nm}^{4}\right)$ & $\beta$ & $p$ & $M_{L}\left(\mathrm{gmol}^{-1} \mathrm{~nm}^{-1}\right)$ & $d_{H}(\mathrm{~nm})$ \\
\hline 200 & - & 5000 & 4 & 12 & 60000 & 52 \\
\hline 100 & - & 350 & 3.5 & 7 & 35000 & 37 \\
\hline 50 & $9.7 \times 10^{31}$ & 60 & 3.5 & 6 & 30000 & 30
\end{tabular}

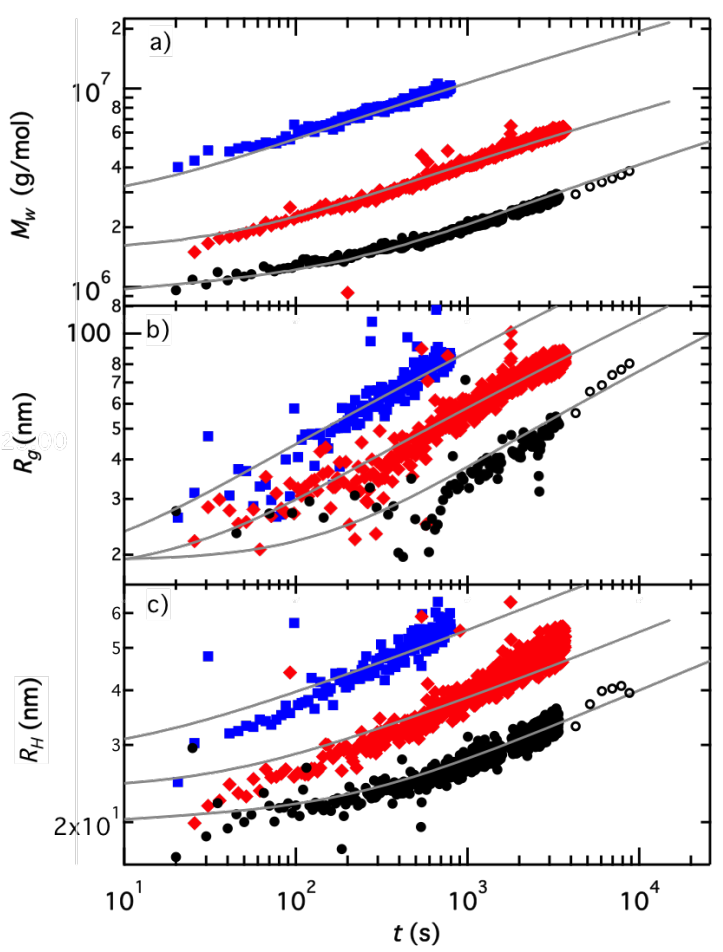

Fig. 5 Time dependent self-assembly of vimentin for different salt concentrations. Data (symbols) along with fits (lines) according to the two step kinetic model (eqs S10-S19 of the Supporting Information). Colours have the same meaning as in Figure $3 . c_{S}=50 \mathrm{mM}(\bullet), 100 \mathrm{mM}(\bullet)$ and $200 \mathrm{mM}(\boldsymbol{\square})$. Hollow symbols correspond to 30 angle sweeps. a) $M_{w}$, b) $R_{g}$, c) $R_{H}$. Fit parameters are collected in Table 2

We find that increasing the concentration of added $\mathrm{KCl}$ in the $c_{S}=50-200 \mathrm{mM}$ range leads to faster elongation kinetics for vimentin filaments but does not alter the assembly mechanism. Modeling of the experimental data with a two step process including ULF formation and filament elongation reveals a length dependence of the rate constant of elongation of $L^{-4}$, which is stronger than suggested by Hill ${ }^{41}$, who predicts a decay of the rate constant of $\sim L^{-2}$. The increase of the rate of filament formation with the salt concentration was captured by a drastic increase of the rate constant of filament elongation. In terms of the kinetic model, this corresponds to an increase of an area surrounding two approaching ends of filaments, which specify the minimum spacial range for successful end-to-end linking. Light scattering and STEM data indicate that the mass per unit length of the filaments increase with increasing added salt concentration. Data for
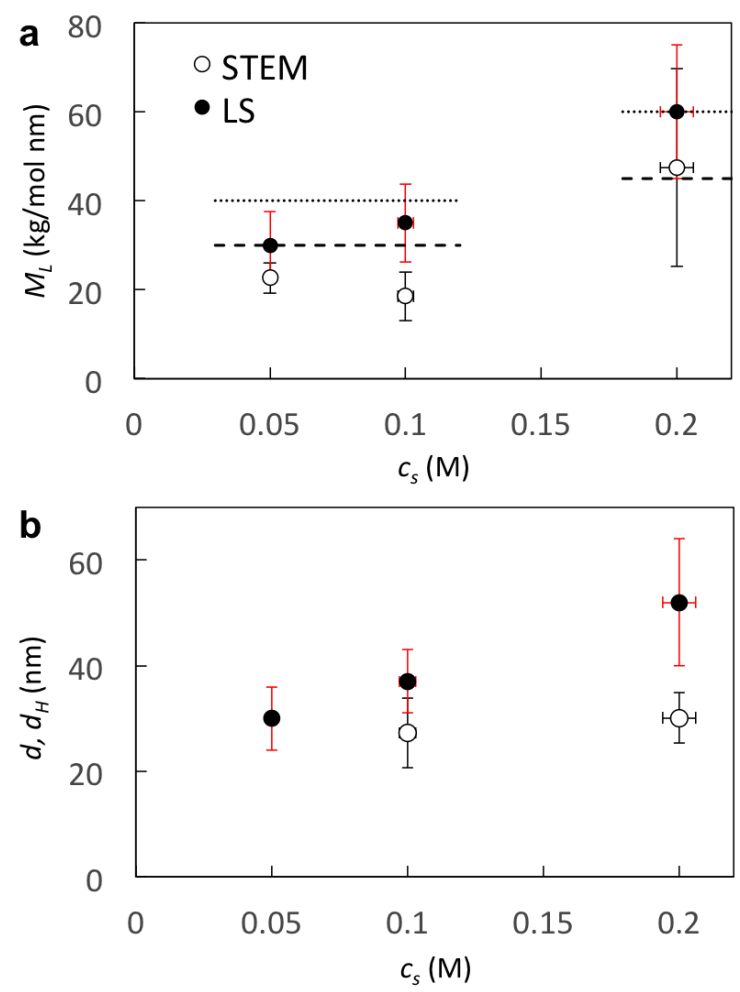

Fig. 6 Structural and hydrodynamic parameters for vimentin filaments as a function of added salt concentration. a) Mass per unit length from STEM and LS data. The lines represent $M_{L}$ for $p=8$ (low salt) and $p=$ 12 (high salt). Dotted lines are calculated using $M_{L}=60 / 43 p M_{L, t e t}$, where $M_{L, t e t} \simeq 3570 \mathrm{gmol}^{-1} \mathrm{~nm}^{-1}$ is the mass per unit length of a tetramer, the factor of 60/43 arises from the partial longitudinal overlap of filaments during end-to-end annealing. Dashed lines use $M_{L}=p M_{L, t e t}$. These two values correspond to the limiting cases of long filaments and ULFs respectively. The difference arises due to partial overlap of filaments during end-to-end annealing. The error for LS data results from the uncertainty in the protein concentration, and hence affects all samples by the same factor, see the text for details. b) Filament width from STEM data $(d, \circ)$ and hydrodynamic cross sectional diameter from LS data $\left(d_{H}\right.$, -). At $c_{S}=50 \mathrm{mM}$ the intensity of the filaments is too low for accurate determination of filament width.

$50 \leq c_{S} / \mathrm{mM} \leq 100$ are consistent with literature reports indicating filaments made up of $p \simeq 8$ laterally assembled tetramers. At $c_{S}=200 \mathrm{mM}$ a higher value of $p \simeq 12$ is observed. Such variation in filament diameters could possibly have physiological impact. Although the overall concentration of potassium in the cytoplasm of eukaryotes is $154 \mathrm{mM}$, locally different concentration may be 
found. These varying concentrations, in turn, may lead to locally varying mechanical properties 10 . Such a mechanism could add yet another cellular "tool" to adjust its mechanical properties. In addition to the salt concentration present in the solution, the type and valence of ions plays a role in assembly and has been studied by electron microscopy, transient electric birefringence and small angle $\mathrm{x}$-ray scattering, especially for the comparison of potassium and magnesium $17 / 46 / 48$. It may be an interesting future approach to systematically study such different conditions using our method and thus access the elongation reaction during assembly. The $\mathrm{pH}$ of the solution, as well, plays an important role for assembly and may be an interesting target for future studies. Furthermore, it may be interesting to investigate other IF protein, such as desmin or keratin, albeit the time scales for assembly may not fit our experimental boundary conditions as well as they do for vimentin: keratin assembles about 100 times faster than vimentin 49 and it is therefore questionable whether the relevant steps can be captured by our technique. With the present study we demonstrated how a thorough and quantitative characterization of the hierarchical assembly of intermediate filament proteins into high-order structures can be performed using SLS and DLS. We believe that the method is well suited to also study other systematically varying conditions, thus helping to shed light on the complex assembly mechanisms of these proteins.

\section{Conflicts of interest}

There are no conflicts to declare.

\section{Acknowledgements}

We thank Harald Herrmann for fruitful discussions, Susanne Bauch for technical assistance, and Sanjib Saha for assistance with the SLS/DLS measurements. Tobacco Mosaic Virus was generously provided by Jean-Luc Pellequer. This work was supported by the German Research Foundation (DFG) in the framework of SFB 755 'Nanoscale Photonic Imaging', project B07 and DFG KO 3572/5-1, as well as the Helmholtz Gemeinschaft in the framework of the Virtual Institute VH-VI-403 'In-Situ Nano-Imaging of Biological and Chemical Processes'. The STEM work was financially supported by the the Netherlands Organisation for Scientific Research (NWO), with a program grant from the Foundation for Fundamental Research on Matter (FOM Program grant nr 143) and a Veni fellowship.

\section{Notes and references}

1 F. Huber, A. Boire, M. P. Lopez and G. H. Koenderink, Current opinion in cell biology, 2015, 32, 39-47.

2 K. Seltmann, A. W. Fritsch, J. A. Käs and T. M. Magin, Proceedings of the National Academy of Sciences, 2013, 110, 1850718512.

3 L. Ramms, G. Fabris, R. Windoffer, N. Schwarz, R. Springer, C. Zhou, J. Lazar, S. Stiefel, N. Hersch, U. Schnakenberg, T. M. Magin, R. Leube, Rudolf E. Merkel and B. Hoffmann, Proceedings of the National Academy of Sciences, 2013, 110, 1851318518.

4 J. Block, V. Schroeder, P. Pawelzyk, N. Willenbacher and S. Köster, Biochimica et Biophysica Acta (BBA)-Molecular Cell
Research, 2015, 1853, 3053-3064.

5 J. Kayser, H. Grabmayr, M. Harasim, H. Herrmann and A. R. Bausch, Soft Matter, 2012, 8, 8873-8879.

6 B. Nöding and S. Köster, Physical Review Letters, 2012, 108, 088101.

7 N. Mücke, L. Kreplak, R. Kirmse, T. Wedig, H. Herrmann, U. Aebi and J. Langowski, Journal of molecular biology, 2004, 335, 1241-1250.

8 M. Schopferer, H. Bär, B. Hochstein, S. Sharma, N. Mücke, H. Herrmann and N. Willenbacher, Journal of molecular biology, 2009, 388, 133-143.

9 L. Kreplak, H. Herrmann and U. Aebi, Biophysical journal, 2008, 94, 2790-2799.

10 J. Block, H. Witt, A. Candelli, E. J. Peterman, G. J. Wuite, A. Janshoff and S. Köster, Physical Review Letters, 2017, 118, 048101.

11 L. Kreplak, H. Bär, J. Leterrier, H. Herrmann and U. Aebi, Journal of molecular biology, 2005, 354, 569-577.

12 H. Herrmann and U. Aebi, Cold Spring Harbor Perspectives in Biology, 2016, 8, a018242.

13 A. A. Chernyatina, D. Guzenko and S. V. Strelkov, Current Opinion in Cell Biology, 2015, 32, 65-72.

14 C. G. Lopez, O. Saldanha, K. Huber and S. Köster, Proceedings of the National Academy of Sciences, 2016, 113, 11152-11157.

15 H. Herrmann, M. Häner, M. Brettel, S. A. Müller, K. N. Goldie, B. Fedtke, A. Lustig, W. W. Franke and U. Aebi, Journal of Molecular Biology, 1996, 264, 933-953.

16 H. Herrmann and U. Aebi, Current Oppinion in Cell Biology, 1998, 8, 177-185.

17 M. E. Brennich, S. Bauch, U. Vainio, T. Wedig, H. Herrmann and S. Köster, Soft Matter, 2014, 10, 2059-2068.

18 N. Mücke, S. Winheim, H. Merlitz, J. Buchholz, J. Langowski and H. Herrmann, PloS one, 2016, 11, e0157451.

19 A. Premchandar, N. Mücke, J. Poznański, T. Wedig, M. KausDrobek, H. Herrmann and M. Dadlez, Journal of Biological Chemistry, 2016, 291, 24931-24950.

20 E. Czeizler, A. Mizera, E. Czeizler, R.-J. Back, J. E. Eriksson and I. Petre, IEEE/ACM Transactions on Computational Biology and Bioinformatics (TCBB), 2012, 9, 885-898.

21 R. Kirmse, S. Portet, N. Mücke, U. Aebi, H. Herrmann and J. Langowski, Journal of Biological Chemistry, 2007, 282, 18563-18572.

22 S. Portet, N. Mücke, R. Kirmse, J. Langowski, M. Beil and H. Herrmann, Langmuir, 2009, 25, 8817-8823.

23 S. Portet, Journal of Theoretical Biology, 2013, 332, $20-29$.

24 A. V. Sokolova, L. Kreplak, T. Wedig, N. Mücke, D. I. Svergun, H. Herrmann, U. Aebi and S. V. Strelkov, Proceeding of the National Academy of Sciences of the United States of America, 2006, 103, 16206-16211.

25 M. E. Brennich, J.-F. Nolting, C. Dammann, B. Nöding, S. Bauch, H. Herrmann, T. Pfohl and S. Köster, Lab on a Chip, 2011, 11, 708-716.

26 O. Saldanha, M. E. Brennich, M. Burghammer, H. Herrmann and S. Köster, Biomicrofluidics, 2016, 10, 024108. 
27 W. Ip, M. K. Hartzer, Y.-Y. S. Pang and R. M. Robson, Journal of Molecular Biology, 1985, 183, 365-375.

28 L. Kreplak, K. Richter, U. Aebi and H. Herrmann, Methods in Cell Biology, 2008, 88, 273-297.

29 S. Köster, D. A. Weitz, R. D. Goldman, U. Aebi and H. Herrmann, Current opinion in cell biology, 2015, 32, 82-91.

30 S. Müller and A. Engel, Micron, 2001, 32, 21-31.

31 A. A. Sousa and R. D. Leapman, Nanoimaging: Methods and Protocols, 2013, 195-207.

32 S. Mallat and S. Zhong, IEEE Transactions on pattern analysis and machine intelligence, 1992, 14, 710-732.

33 B. H. Zimm, The Journal of Chemical Physics, 1948, 16, 10991116.

34 B. H. Zimm, The Journal of Chemical Physics, 1948, 16, 10931099.

35 B. J. Frisken, Applied Optics, 2001, 40, 4087-4091.

36 A. G. Mailer, P. S. Clegg and P. N. Pusey, Journal of Physics: Condensed Matter, 2015, 27, 145102.

37 P. Hassan and S. Kulshreshtha, Journal of Colloid and Interface Science, 2006, 300, 744-748.

38 M. Schmidt, Macromolecules, 1984, 17, 553-560.

39 W. Burchard, Light Scattering from Polymers, Springer, 1983, pp. 1-124.

40 R. Carrotta, M. Manno, D. Bulone, V. Martorana and P. L.
San Biagio, Journal of Biological Chemistry, 2005, 280, 30001-30008.

41 T. L. Hill, Biophysical Journal, 1983, 44, 285.

42 S. Saha, S. Springer, M. E. Schweinefuß, D. Pontoni, M. Wiebcke and K. Huber, Crystal Growth \& Design, 2016, 16, 2002-2010.

43 B. Nöding, H. Herrmann and S. Köster, Biophysical Journal, 2014, 107, 2923-2931.

44 A. V. Sokolova, L. Kreplak, T. Wedig, N. Mücke, D. I. Svergun, H. Herrmann, U. Aebi and S. V. Strelkov, Proceedings of the National Academy of Sciences, 2006, 103, 16206-16211.

45 H. Lodish, A. Berk, S. L. Zipursky, P. Matsudaira, D. Baltimore and J. Darnell, Molecular Cell biology 4th edition, W. H. Freeman, 2000.

46 I. Hofmann, H. Herrmann and W. Franke, European journal of cell biology, 1991, 56, 328-341.

47 H. Herrmann, M. Häner, M. Brettel, N.-O. Ku and U. Aebi, Journal of molecular biology, 1999, 286, 1403-1420.

48 M. Kooijman, M. Bloemendal, P. Traub, R. van Grondelle and H. van Amerongen, Journal of Biological Chemistry, 1995, 270, 2931-2937.

49 T. Lichtenstern, N. Mücke, U. Aebi, M. Mauermann and H. Herrmann, Journal of structural biology, 2012, 177, 5462. 